\title{
Médecins suisses en route pour Aceh, la région la plus touchée par la catastrophe dans le Nord de Sumatra
}

\author{
O. Seeger
}

Les premières délégations de médecins suisses se sont envolées pour Medan. Ils emportent avec eux tout l'équipement de premier secours nécessaire. Ils aideront et participeront à l'installation d'équipements médicaux de base.

Correspondance:

Oliver Seeger

PanEco

Chileweg 5

CH-8415 Berg am Irchel

E-mail: mail@paneco.ch
L'appel lancé dans toute la Suisse par la fondation PanEco, en accord avec l'Association Suisse des médecins-assistant(e)s et chef(fe)s de clinique (section de Zurich), pour trouver des médecins et du personnel soignant qualifié a suscité un vif intérêt. Jusqu'à présent, non seulement des Suisses ont proposé leur aide, mais également des personnes des Pays-Bas, de la Belgique, de l'Australie, de la Thailande et de Singapour.

Le médecin Sofyan Tan qui travaille pour l'ONG environnementale YEL a communiqué que 500 médecins et 700 personnes soignantes, ainsi que des médicaments, étaient requis dans la région la plus touchée du Nord de Sumatra. Le Dr. Ian Singleton, directeur scientifique de SCOP, mentionne également dans un rapport les conditions horribles dans lesquelles les survivants doivent recevoir les soins médicaux. Il signale des villages entièrement détruits et dépeuplés, et des régions non seulement vides d'humains mais quasiment balayées par le raz de marée. Il semble que de mémoire d'homme, ce soit la plus grande catastrophe que l'on n'ait jamais vue.

Les médecins, les personnes soignantes et les équipements médicaux seront transportés à Sumatra par PanEco qui travaille depuis longtemps à Sumatra et particulièrement en Aceh avec son partenaire local, l'organisation non gouvernementale YEL. Avant d'être intégrés dans les équipes des ONG, les médecins et soignants seront reçus à Medan dans le centre de coordination dirigé par Yel, qui envoie les équipes là où seront créées les bases sanitaires et médicales, et distribue les médicaments.

Tous ces efforts ne représentent qu'une goutte d'eau dans la mer! Ce n'est pas la volonté d'entraide qui manque mais plutôt les possibilités de participer concrètement à cette aide humanitaire si urgente et nécessaire.

Médecins, personnel soignant, médicaments et équipements sont toujours nécessaires pour soigner et sauver les victimes, et le temps passe. 